\title{
INHIBITORY EFFECTS ON TUMOR NECROSIS FACTOR ALPHA AND INTERLEUKIN 12 USING CLOBETASOL PROPIONATE LOADED TEA TREE OIL NANOEMULSION GEL ON ANIMAL MODEL
}

\author{
MD SARFARAZ ALAM ${ }^{1 *}$, MOHAMAMMAD DAUD ALI ${ }^{2}$, MD SALAHUDDIN ANSARI ${ }^{1}$, PANKAJ SHARMA ${ }^{1}$
}

${ }^{1}$ Department of Pacific College of Pharmacy, Pacific Academy of Higher Education and Research University, Udaipur, Rajasthan, India. ${ }^{2}$ Department of Pharmacy, Mohammed Al-mana College of Health Science, Dammam, Kingdom of Saudi Arabia. Email: mdalam814160@gmail.com

Received: 22 January 2018, Revised and Accepted: 20 February 2018

\section{ABSTRACT}

Objective: The main objective of our study is to explore anti-inflammatory activity at its molecular level like tumor necrosis factor alpha (TNF- $\alpha$ ), interleukin 12 (IL-12) expression, and histopathological study.

Methods: As per solubility/miscibility of clobetasol propionate (CP) with tea tree oil (TTO), surfactant and cosurfactant ( $\left.\mathrm{S}_{\text {mix }}\right)$, and water in a ratio of oil: $S_{\text {mix }}$ :water (15:35:50) taken in milliliter for the preparation of nanoemulsion. Induced allergic contact dermatitis (ACD) with dinitrofluorobenzene (DNFB) was used for the study. TNF- $\alpha$ and interleukin 12 (IL-12) were estimated with rabbit antimouse TNF- $\alpha$ and rat antimouse IL-12 antibodies in $1 \%$ of bovine serum albumin in phosphate buffer.

Results: Topical application of CP loaded nanoemulsion gel inhibits ear inflammation and erythema in DNFB-induced ACD in mice and significantly reduces the intracellular edema and infiltration with inflammatory mediator cells involving of mononuclear cells and neutrophils. CP loaded nanoemulsion gel reduces expression of protein level of TNF- $\alpha$ and IL-12.

Conclusion: CP loaded nanoemulsion gel confirmed that anti-inflammatory effects showed more rapidly than the placebo and marketed gel preparation. However, the animals treated with placebo nanoemulsion gel showed a somehow comparable reduction of their inflammation during treatment compared with the marketed gel. This effect may be due to anti-inflammatory effect of TTO. This result suggested that anti-inflammatory activity of placebo nanoemulsion gel may be due to TTO present in nanoemulsion as vehicle.

Keywords: Nanoemulsion, Tea tree oil, Tumor necrosis factor alpha, Interleukin 12.

(c) 2018 The Authors. Published by Innovare Academic Sciences Pvt Ltd. This is an open access article under the CC BY license (http://creativecommons. org/licenses/by/4. 0/) DOI: http://dx.doi.org/10.22159/ajpcr.2018.v11i6.24888

\section{INTRODUCTION}

Psoriasis is an autoimmune inflammatory skin condition in which abnormal epidermal differentiation and hyperproliferation occurs. Worldwide 2-3\% of population suffering from such kind of skin disease [1]. The exact cause of this disease is still not clear but it is assumed that it may be mainly due to the activation and migration of $\mathrm{T}$ cells to the dermis which triggering the release of cytokines tumor necrosis factor alpha (TNF- $\alpha$ ) ultimately lead to the inflammatory process and the rapid proliferation of skin cells. TNF- $\alpha$ is considered to be central dogma of inflammatory cascade $[2,3]$. Therefore, the drug which has potential to inhibit TNF- $\alpha$ very useful for the treatment of psoriasis. There are many risk factors which can be responsible for psoriasis such as emotional as well as mental stress condition for a long time, injury to skin, infections up to systemic level, certain medication that may trigger, and gastrointestinal disorder. Numerous kinds of psoriatic conditions have been documented such as plaque type of psoriasis, psoriatic arthritis, scalp psoriasis, flexural psoriasis, guttate psoriasis, and pustular psoriasis [4]. All these types of psoriasis can be identifying by clinical test such as biopsy of skin and antigenantibody reaction. Currently, the drug prescribing for the treatment of psoriasis can either modulate the immune system or try to normalize the hyperactive differentiation process of psoriatic keratinocytes of the skin. On the basis of the psoriatic condition, its localization, extent, and degree of severity there are numerous modes of the treatment regimens utilizing for psoriatic condition such as topical application of the dosages forms, phototherapy, systemic therapy, and also drugs used that comes under chemotherapy which can be a potential approaches to control the psoriatic condition. Plaque types of psoriatic condition constitute about $80 \%$ of people who develop psoriasis. Physicians should find out suitable idea of treatment and make it clear to the patient that the main objective of treatment is control of the psoriatic condition. $<5-10 \%$ of the body surface area is defined as mild to moderate psoriasis [5]. Majority of the patients comes under this category. Therefore, physician given preference to the topical preparation for such kind of patients. Another reason behind these approaches to keep patients on minimum side effects as it is recognized that drugs used for psoriatic condition may leads to numerous adverse effects. It is also true that patient needs continuous medication to control psoriatic condition. Currently, the drugs used for the treatments are corticosteroids, salicylic acid, and emollients. Other drugs include Vitamin D analogs such as calcipotriene and calcitriol, tar, and topical retinoids in the form of conventional cream, lotion, ointments, and other preparation also. However, the use of glucocorticoids like CP in the form of conventional dosages forms limit their clinical utility because of the poor availability of the drug in the dipper site of the skin where it has to shows action [6,7]. The limited permeability of the drug through the skin is mainly because of the barrier function of the hyperkeratinized cells of the skin in psoriatic condition. Due to this condition, our current work focused on novel dosages form to deliver the drug to the dipper site of skin and remove the hypercriticized cells from topmost layer of the skin. In view of that salicylic acids added to the nanoemulsion during gelling to remove hyperkeratinized cell from superficial layer of skin. The dosages forms also provide a sufficient increase in drug penetration into the skin, without any countable functional and histological change in the skin structure [8]. If any such minor alteration was seen, it must be reversible after discontinuation of therapy. In the current decade, different types of lipid-based formulations formulated for the improvement of permeability and bioavailability of less water-soluble and highly lipid-soluble active drugs, but most of them not fulfill all the desire 
parameter related to safe delivery of drug for the psoriasis treatment. Most of the topical preparation used currently, they contains chemical penetration enhancers to increase drug availability to the dipper site of the skin. However, the problem faced with the use of these chemical enhancers in topical preparation may leads to irritation, redness on skin, stinging, or itching sensation to the skin when applied for a long term. Therefore, naturally derived tea tree oil (TTO) selected for the current work which act as a carrier for the drug for nanoemulsion as well as penetration enhancer for the formulation. This oil has potential to reduce inflammation. The antiseptic activity of TTO is also sometimes may become beneficial because psoriasis may be trigger due to some microbial infection [9]. One of the most novel formulations for the increase of skin permeation of drugs is nanoemulsion. The added advantages of nanoemulsions in terms of low irritation to skin, strong permeation ability of drug to the dipper site of skin, and high drug loading capacity leads to superior over the other dosages forms for topical application $[10,11]$. The main objective of our study is to explore anti-inflammatory activity at its molecular level such as TNF- $\alpha$, IL-12 expression, and histopathological study.

\section{MATERIALS AND METHODS}

Materials

Drug sample, i.e., clobetasol propionate (CP) was gifted as a gift sample from Ranbaxy Research Laboratory (Gurgaon, India). TTO was gifted as a gift sample from Natural Aroma Products Pvt. Ltd., New Delhi, India. PEG 200, PEG 400, Tween 40, Tween 20, Tween 60, and ethanol were procured from Merck (Merck, India). Plurol Oleique, Transcutol P, Labrafac, and Labrasol were obtained as gift sample on request from Gattefosse (India). CMC, HPMC, sodium alginate, Carbopol 934, and salicylic acid were gifted from S.D. Fine chemicals, India. All other chemicals were of generally recognized as safe categories.

Formulation of nanoemulsion gel (A2) containing salicylic acid Pure nanoemulsion is not suitable for topical application as it exhibits low viscosity. The viscosity needs to be increased by incorporating some thickening agents, which may contribute to change the appearance and texture of the system. Another thing is that it may influence the drug release from the gel. The ethyl cellulose, xanthan gum, sodium alginate, Carbopol 934, and HPMC are currently used as a gelling agent to increase the thickness of nanoemulsion up to the desire viscosity. Suitability of polymer for the preparation of gel is usually on the basis of the characteristic of external phase nanoemulsion either oil or water. $\mathrm{CP}$ nanoemulsion is a type of oil in water $(\mathrm{o} / \mathrm{w})$ nanoemulsion: Sodium alginate, ethyl cellulose, HPMC, and Carbopol 934 were selected for the preparation of nanoemulsion gel. Preservatives and other substance for $\mathrm{pH}$ adjustment used as per need [12].

\section{Animals}

Mice aged 8 to 10 weeks of either sex, weighing 16-18 g, were received from university animal house. The animals were kept under conventional conditions and supplied with rodent chow and water ad libitum. The animal study protocol was approved by our university.

Induction of allergic contact dermatitis (ACD) with dinitrofluorobenzene (DNFB)

ACD was induced by the method that back skin of each mouse was painted with $100 \mu \mathrm{L}$ of $0.5 \%$ of DNFB (Sigma Aldrich, MO, USA) in 4:1 (acetone:olive oil). 5 days later, the mice were challenged on both sides of the ears with $20 \mu \mathrm{L}$ of $0.2 \%$ of DNFB solution. The animals were rechallenged after 2 days to yield extensive disease. 1 day after, the rechallenge the animals began to be treated with the test compounds. The degree of ear inflammation and erythema was used to measure of ACD. Erythema scored measured on a scale of $0-4$. Where, 0 was absent and 4 was severe pinkish-red color of the ear. Ear thickness was measured before application (challenge) and then pre- and post-application [13].

\section{Treatments}

Groups of 15-30 animals were treated with a topical application of approximately $100 \mathrm{mg}$ of the following test compounds, once a day for
4 days: Placebo nanoemulsion gel, $0.05 \%$ CP loaded nanoemulsion gel, and $0.05 \%$ marketed formulation gel.

\section{Histopathology and immunohistochemistry}

Previously, formalin fixed as well as paraffin-embedded sections of the mouse ears was used for the study. $5 \mu \mathrm{m}$ thick sections were stained using hematoxylin and eosin as per standard methods. All the work was examined by an expert veterinary pathophysiologist, but they were unaware of the treatment of the samples which was going to be examined. Histopathological changes in each of the three layers such as epidermis, dermis, and subcutis were ranked on a scale of $0-4$ as proposed by Wang et al. [14]

For the evaluation of immunohistochemistry, a universal streptavidin/biotin detection system (Pittsburgh, USA) was used. Before the study, removal of wax and hydration carried out for the sections which were undergoes for the study. Then, the sections were undergoes treatment with protein blocking agent so that to reduce non-specific binding of antibodies. The sections were then incubated for specified time with rabbit antimouse TNF- $\alpha$ and rat antimouse IL- 12 antibodies (Biosource International, USA) in 1\% of bovine serum albumin in phosphate buffer. Incubation with the secondary antibodies, streptavidin/biotin, and chromogenic detection of peroxidase was done as per the supplier's protocol as mentioned in kits. Then, sections undergo for counterstaining and removal of extra water. Now, the dehydrated sections were mounted and examined. The intensity of the immunoperoxidase staining was ranked as designated by Arican et al. [15].

The expression of cytokines in sections was rated as: 0 for no staining in the Section 1 for $<25 \%$ of the sections were positive, 2 for $25-50 \%$ of the section, 3 for $50-75 \%$ of the section, and 4 for more than $75 \%$ of the section with major staining [16].

\section{Statistics}

The statistical significance of the difference in mean ear erythema, thickness, histopathological changes, number of apoptotic cells, and the expression of cytokines was determined using Tukey-Kramer's multiple comparison tests following one-way analysis of variance. Data were expressed as mean \pm SEM. $\mathrm{p} \leq 0.05$ was considered statistically significant.

\section{RESULTS AND DISCUSSIONS}

Formulation of nanoemulsion gel containing salicylic acid Nanoemulsion gel prepared by taking two grams of Carbopol 934 and five grams of salicylic acid were added in $87.68 \mathrm{ml}$ of the water and stirrer with the help of magnetic stirred till homogenous mixture was obtained. After that, drop by drop added prepared nanoemulsion of $12.32 \mathrm{ml}$ to make it volume of $100 \mathrm{ml}$ till homogeneous mixture obtained. As per solubility/miscibility of CP with TTO, surfactant and cosurfactant $\left(\mathrm{S}_{\text {mix }}\right)$, and water in a ratio of oil: $\mathrm{S}_{\text {mix }}$ :water $(15: 35: 50)$ taken in milliliter for the preparation of nanoemulsion. $2 \mathrm{ml}$ of TTO sufficient to dissolve $50 \mathrm{mg}$ drug in $100 \mathrm{ml}$ of total preparation; therefore, total volume of nanoemulsion was $12.32 \mathrm{ml}$.

\section{Effects of CP loaded nanoemulsion gel on DNFB-induced ACD in} mice

Within $24 \mathrm{~h}$ of rechallenge with DNFB, all mice developed experimental signs and symptoms of erythema as well as edema. The topical treatments of $0.05 \% \mathrm{CP}$ loaded nanoemulsion significantly reduced ear thickness after 1 day and erythema after 3 days of treatment (Fig. 1a and b). When it compared with placebo nanoemulsion and marketed preparation.. Placebo nanoemulsion also reduced the ear thickness after 1 day and the erythema after 2 days of treatment (Fig. 1a and b). After 4 days of treatment, no significant differences among these three active treatments were found. Significant reduction of body weight after 3 days of treatment was observed in the group of animals treated with CP loaded nanoemulsion compared with the other treatment groups so that by day 4 average loss in this group was about $13 \%$ of body 
weight and mice showed piloerection, lethargy, and a clearly abnormal appearance.

Histopathologically, the reductions in inflammation in the groups treated with placebo nanoemulsion gel, CP loaded nanoemulsion gel, and marketed gel were compared with the no treatment groups (Fig. 2). Mark differences among the effective treatments were observed. After 4 days of treatment, most of the sections from the active treatment groups showed normal epidermis and significantly reduced inflammatory cells in case of CP loaded nanoemulsion, whereas edema and inflammatory cell infiltration were seen in the sections from ears treated with placebo nanoemulsion gel or untreated (Fig. 3).

Effects of CP loaded nanoemulsion gel on cytokines protein expression determined by immunohistochemistry

Both IL-12 and TNF- $\alpha$ were upregulated after rechallenge with DNFB. As seen in Figs. 4 and 5, the positive staining for both IL-12 and TNF- $\alpha$ was markedly reduced in the groups treated with CP loaded nanoemulsion gel, with respect to placebo and marketed gel after 4 days of treatment. When the grading of the intensity of the staining for IL-12 and TNF- $\alpha$, was analyzed, CP loaded nanoemulsion gel significantly reduced the protein expression of IL-12 and TNF- $\alpha$ with respect to placebo and marketed gel (Fig. 6) with significant differences among them

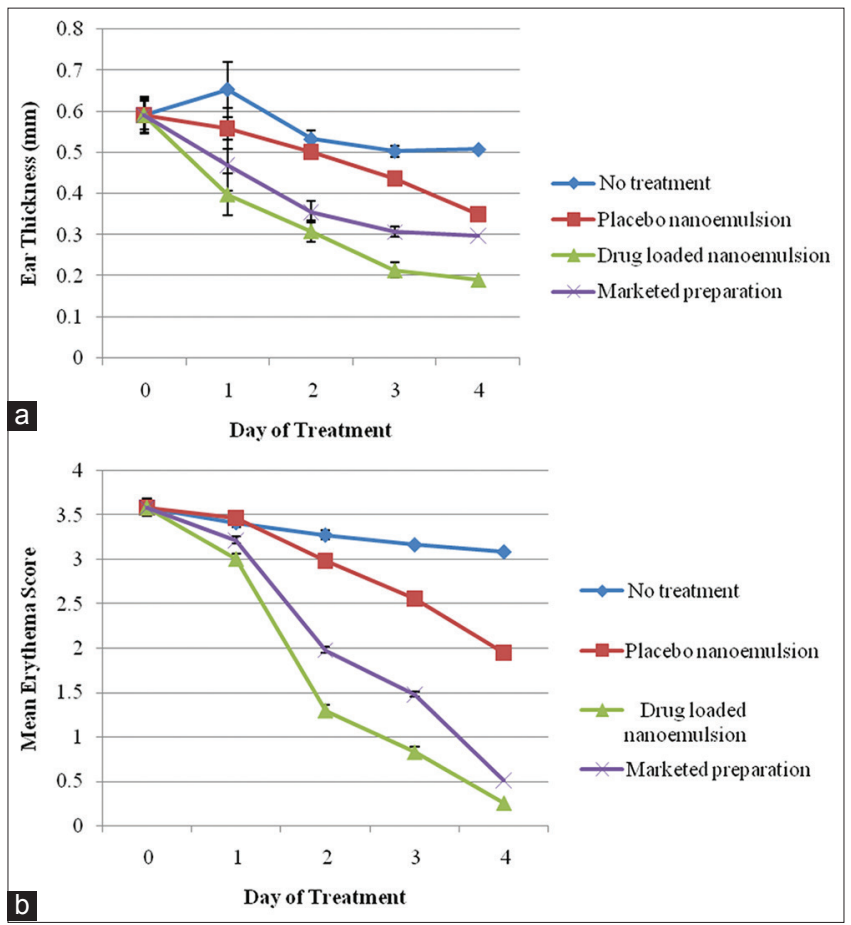

Fig. 1: (a) Ear thickness scores as a function of treatment in mice with allergic contact dermatitis (ACD). (b) Erythema scores as a function of treatment in mice with ACD. Values are mean \pm standard error mean, $n=15-30$

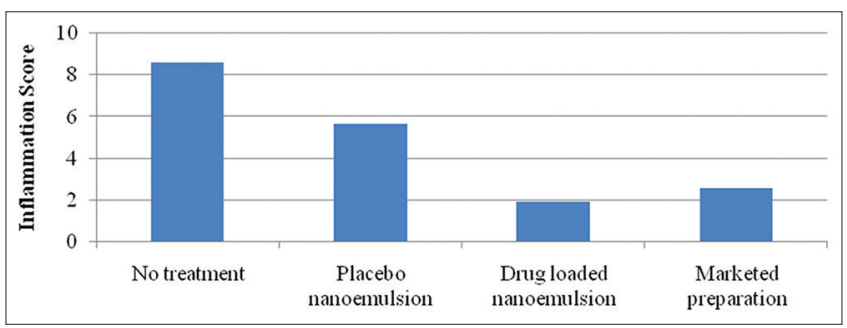

Fig. 2: Histopathological inflammation scores of allergic contact dermatitis in mice after 4 days of treatment, $n=15$
The present study demonstrates that topical application of CP loaded nanoemulsion gel inhibits ear inflammation and erythema in DNFBinduced ACD in mice and significantly reduces the intracellular edema and infiltration with inflammatory mediator cells involving of mononuclear cells and neutrophils. In comparison to placebo and
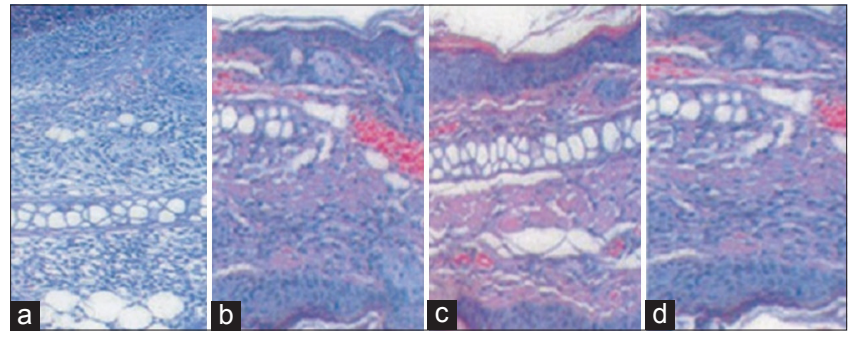

Fig. 3: Clobetasol propionate (CP) loaded nanoemulsion gel reduces inflammation and inflammatory infiltration in the allergic contact dermatitis in mice ears. Hematoxylin and eosinstained sections of ears after 4 days of treatment. (a-d) Ears treated with no treatment, placebo gel, marketed gel, and CP loaded nanoemulsion gel, respectively. The sections $\mathrm{D}$, showed normal epidermis, decreased edema and/or few inflammatory cells

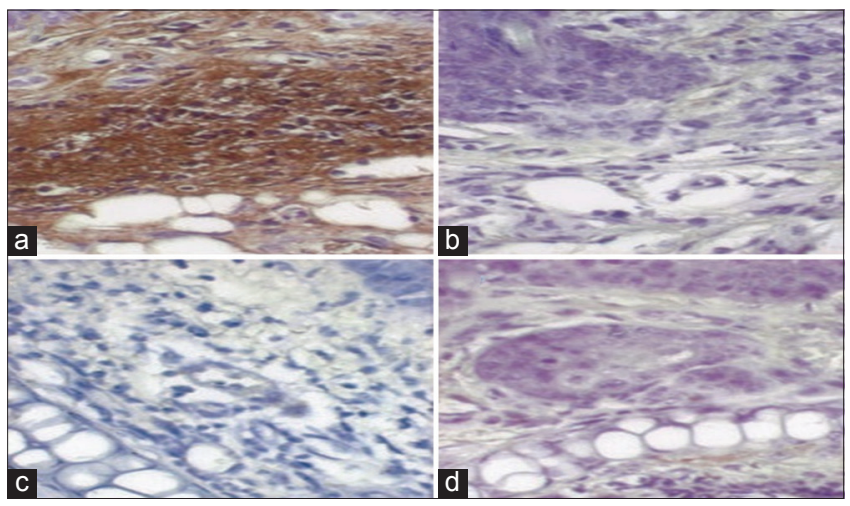

Fig. 4: Clobetasol propionate (CP) loaded nanoemulsion gel reduces protein expression of interleukin (IL)-12 more in compression to other group. Immunohistochemistry of representative sections of mice ears stained with antibodies to IL12. After 4 days treatment with no treatment (a), placebo gel (b), marketed gel (c), and CP loaded nanoemulsion gel (d)

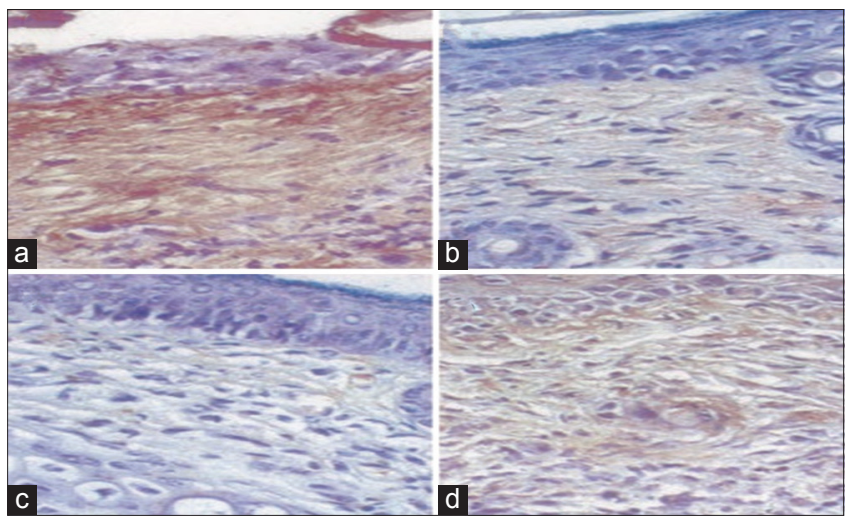

Fig. 5: Clobetasol propionate (CP) loaded nanoemulsion gel reduces protein expression of tumor necrosis factor alpha (TNF- $\alpha$ ). Immunohistochemistry of representative sections of mice ears stained with antibodies to tumor necrosis factor (TNF- $\alpha$ ) after treatment with no treatment (a), placebo gel (b), marketed gel (c), and CP loaded nanoemulsion gel (d) 


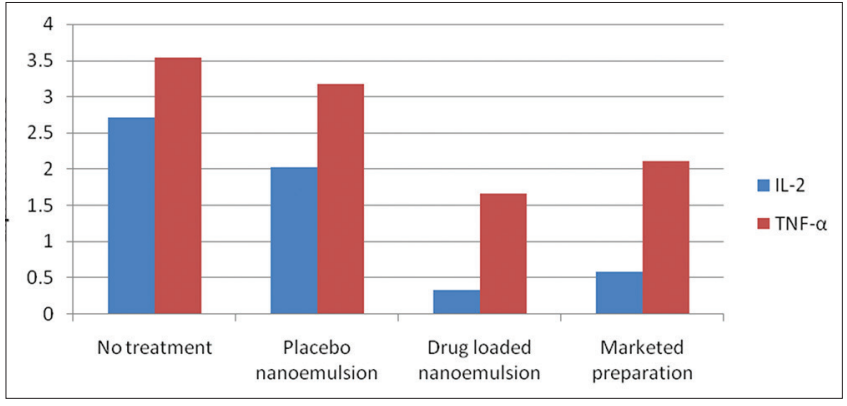

Fig. 6: Clobetasol propionate (CP) loaded nanoemulsion gel reduces expression of protein level of interleukin (IL)-12 (a) and tumor necrosis factor alpha (b). Semi-quantitative assessment of staining of IL-12 and TNF- $\alpha, n=15$

marketed gel preparation, the CP loaded nanoemulsion gel showed more anti-inflammatory action.

\section{CONCLUSION}

In this study, CP loaded nanoemulsion gel confirmed that antiinflammatory effects showed more rapidly than the placebo and marketed gel preparation. However, the animals treated with placebo nanoemulsion gel showed a somehow comparable reduction of their body weight during treatment compared with the marketed gel. This effect may be due to anti-inflammatory effect of TTO. This result suggested that anti-inflammatory activity of placebo nanoemulsion gel may be due to TTO present in nanoemulsion as vehicle.

\section{AUTHOR'S CONTRIBUTIONS}

Mr. Md Sarfaraz Alam contributed in performing the experiment and data compilation. Mr. Mohammad Daud Ali contributed in performing data analysis and data interpretation. Md. Salahuddin Ansari was involved in critical revision of article. Dr. Pankaj Sharma given final approval of the version for publication.

\section{ACKNOWLEDGMENT}

Authors are thankful to the Pacific Academy of Higher Education and Research University, Udaipur, Rajasthan, India-313024, for providing all facilities.

\section{CONFLICTS OF INTEREST}

The authors declare that they have no conflicts of interest.

\section{REFERENCES}

1. Prakash V. Terpenoids as source of anti-inflammatory compounds. Asian J Pharm Clin Res 2017;10:68-76.

2. Baboota S, Alam MS, Sharma S, Sahni JK, Kumar A, Ali J. Nanocarrier-based hydrogel of betamethasone dipropionate and salicylic acid for treatment of psoriasis. Int J Pharm Investig 2011;1:139-47.

3. Gupta G, Sharma P, Kumar P, Sharma R. Scope of inflammatory markers in subclinical hypothyroidism. Asian J Pharm Clin Res 2015;8:24-7.

4. Gizaway S, Fadel M, Mourad B, Elnaby F. Betamethasone dipropionate gel for treatment of localized plaque psoriasis. Int J Pharm Pharm Sci 2017;9:173-82.

5. Thomas CL, Finlay AY. The 'handprint' approximates to $1 \%$ of the total body surface area whereas the 'palm minus the fingers' does not. Br J Dermatol 2007; 157:1080.

6. Fontana MC, Rezer JF, Coradini K, Leal DB, Beck RC. Improved efficacy in the treatment of contact dermatitis in rats by a dermatological nanomedicine containing Clobetasol propionate. Eur J Pharm Bio Pharm 2011;79:241-9.

7. Alam MS, Baboota S, Ali MS, Ali M, Alam N, Alam MI, et al. Accelerated stability testing of betamethasone dipropionate. Int J Pharm Pharm Sci 2012;4:371-4.

8. Kahlweit M, Strey R, Haase D, Kunieda H, Schmeling T, Faulhaber B, et al. Influence of microemulsions on cutaneous drug delivery. Adv Drug Del Rev 2002;54 Suppl 1:S77-98.

9. Pazyar N, Yaghoobi R. Suppression of inflammatory reactions by terpinen-4-ol, a main constituent of tea tree oil, in a murine model of oral candidiasis and its suppressive activity to cytokine production of macrophages in vitro. Pharm Soc Japan Biol Pharm Bull 2013;36:838- 44 .

10. Ramesh R, Boinpally S, Gopinath D, Pratap KA, Srinivasu P, Bhaskara R. Microemulsion as a novel approach in topical delivery of drug. Int J Pharm 2004;274:185-90.

11. Vyas SP, Khar RK. Submicronemulsions. In: Targeted and Controlled Drug Delivery. $1^{\text {st }}$ ed. New Delhi: CBS Publishers; 2002. p. 280-302.

12. Su R, Yang L, Wang Y, Yu S, Guo Y, Deng J, et al. Formulation, development, and optimization of a novel octyldodecanol-based nanoemulsion for transdermal delivery of ceramide IIIB. Int J Nanomed 2017;12:5203-21.

13. Park DK, Lee YG, Park HJ. Extract of Rhus verniciflua bark suppresses 2, 4-dinitrofluorobenzene-induced allergic contact dermatitis. Evid Based Complement Alternat Med 2013;2013:879696.

14. Wang XM, Lu Y, Wu LY, Yu SG, Zhao BX, Hu HY, et al. Moxibustion inhibits interleukin-12 and tumor necrosis factor alpha and modulates intestinal flora in rat with ulcerative colitis. World J Gastroenterol 2012;18:6819-28.

15. Arican O, Aral M, Sasmaz S, Ciragil P. Serum levels of TNF- $\alpha$, IFN- $\gamma$, IL-6, IL-8, IL-12, IL-17, and IL-18 in patients with active psoriasis and correlation with disease severity. Mediators Inflamm 2005;5:273-9.

16. Mesa-Tejada R, Pascal RR, Fenoglio CM. Immunoperoxidase: A sensitive immunohistochemical technique as a "special stain" in the diagnostic pathology laboratory. Hum Pathol 1977;8:313-20. 